\title{
Estimating Surface Characteristics and Extracting Features from Polarisation
}

\author{
Lichi Zhang, Edwin R. Hancock ${ }^{\star}$, and Jing Wu \\ Department of Computer Science, University of York, UK \\ School of Computer Science and Informatics, Cardiff University, UK
}

\begin{abstract}
In this paper we develop a practical method for estimating shape, color and reflectance using only three images taken under polarised light. We develop a novel and practical framework to optimise the estimates and eliminate the redundant information, then investigate three different methods to compare their class discriminating capacities. We present experiment to demonstrate the validity of the proposed method for a database of fruit objects from 5 different classes, and we show that the proposed method is capable of accurately extracting the features of the input examples. The framework can further be applied in a variety fields of computer vision and pattern recognition domains including object recognition and classification.
\end{abstract}

\section{Introduction}

Accurately estimating and reproducing surface appearance is a task of pivotal importance in computer vision and graphics. Applications include object recognition and classification, and image rendering. The appearance of surfaces is determined by shape, color and reflectance [1. These intrinsic surface properties are independent of each other and affect the observed image intensity in a complicated way. Therefore a robust way of simultaneously estimating these surface properties is required for successful object recognition.

There have been a number of attempts in the literature aimed at accurately measuring surface characteristics. However existing methods are limited by their requirement of high cost measurement systems, and a large number of input images. BRDF was firstly introduced in [2. The direct measurement of the reflectance function requires a gonioreflectometer [3], which is both expensive and cumbersome to use. Other available methods use complicated devices such as light stages and geometric domes to build reflectance functions from image intensity variations under different light source directions 4. Recently Ma et al. 5] presented a method to estimate surface normal maps of an object using four spherical gradient illumination patterns from either diffuse or specular reflectance components. The technique relies on structured light, and hence adds scanning time and system complexity to the overloads.

^ Edwin Hancock was supported by a Royal Society Wolfson Research Merit Award. 
Polarisation has proven to be an effective method in the analysis of light reflection in computer vision. Its applications are in reflectance component separation [6] and surface normal estimation. There have been a number of attempts in the literature aimed at surface orientation estimation of objects using polarisation, where the incident light is unpolarised. The specular and diffuse reflections from the objects become partially polarised, and their values analysed by placing a linear polariser in front of the camera and rotating its orientation. Such effects can be exploited for shape recovery using the Fresnel theory, which was used by Wolff and Boult to describe the direct reflection of electromagnetic waves with the given polarisation state of the incident light [7. This leads to a means of surface normal estimation since the zenith angle of the reflected or re-emitted light is constrained by the degree of polarisation, and the azimuth angle is constrained by the phase angle.

In this paper we introduce a novel statistical framework for simultaneously obtaining shape, texture and reflectance properties from a single view using the theory of polarisation. We commence by acquiring the polarised images under retro-reflection settings, and separate the reflectance components by applying the method of blind source separation (BSS) following the work of Zhang et al. [8]. Then we optimise the estimates and eliminate redundant information. The estimates are converted into long vectors which is used for statistical feature extraction.

We also apply three statistical methods to the data for feature extraction. These are the tradiation method of principal component analysis (PCA), and the improved approaches which includes weight map, that are weighted PCA (WPCA) and supervised weighted PCA (SWPCA). The weight map indicates the importance of different locations in discriminating objects, thus the accuracy of results produced by feature extraction methods can be improved. The methods are developed based on the works of Wu et al. 9. In summary, the novel contributions of this paper are:

1. We provide a novel framework which estimates shape, color and reflectance information using polarisation measurements, that only requires three input images for each object and low-cost devices.

2. We develop optimization methods which eliminate redundant information from the reflectance estimates, so that the feature extraction approaches can produce results accurately and efficiently.

3. We use three statistical methods for feature extraction, which are PCA, WPCA and SWPCA. All the approaches are also optimised to be applied in multi-class recognition.

\section{Modeling Surface Characteristics}

In this section we present the methods used for estimating reflectance, shape and color properties from polarised images. When light arrives at a surface, part of it undergoes isotropic subsurface scattering before being re-emitted which is 
denoted as diffuse reflection. The remainder is reflected in a specular manner. According to the dichromatic model [10, for every pixel in the images its intensity $I$ is decided by two reflectance components which are diffuse $I_{d}$ and specular $I_{s}$ by $I=I_{d}+I_{s}$. The process of detailed reflectance estimation can be simplified if the specular and diffuse components are separated beforehand. Using the blind source separation (BSS) method introduced in [8], we obtain the separated reflectance components from the polarised images.

Reflectance and Shape Estimation: We apply two reflectance models to reflectance measurements. They are Lambertian model used for diffuse component, and Torrance-Sparrow (T-S) 11] for specular component estimation. The two models are simplified under retro-reflection and isotropy. Let the specular and diffuse reflectance models be $R_{s}\left(\theta_{s}, E_{s}\right)$ and $R_{d}\left(\theta_{d}, E_{d}\right)$ respectively, and let $E_{s}$ and $E_{d}$ be their parameter value sets. The estimated surface normal zenith angles for the two models are $\theta_{s}$ and $\theta_{d}$. Associated with each model is a scaling coefficient, denoted by scaler $k_{s}$ and $k_{d}$. We numerically invert the reflectance function to recover the surface zenith angle using

$$
\begin{aligned}
& \theta_{s}=R_{s}^{-1}\left(I_{s} / k_{s}, E_{s}\right)=R_{s}^{-1}\left(I_{s}^{\prime}, E_{s}\right), \\
& \theta_{d}=R_{d}^{-1}\left(I_{d} / k_{d}, E_{d}\right)=R_{d}^{-1}\left(I_{d}^{\prime}, E_{d}\right) .
\end{aligned}
$$

We compute $k_{s}$ and $k_{d}$ so that the two components $I_{s}^{\prime}=I_{s} / k_{s}$ and $I_{d}^{\prime}=I_{d} / k_{d}$ are normalized. Since they correspond to the same image location, $\theta_{s}$ and $\theta_{d}$ should be identical. As $I_{s}$ and $I_{d}$ are known, the parameter values can be found when the distributions of $\theta_{s}$ and $\theta_{d}$ are closest to each other. Because of its rapid (quadratic) convergence we use Newton's method to estimate the parameters $E_{s}$ and $E_{d}$, using a mutual information criterion (M) for the distributions of $\theta_{d}$ and $\theta_{s}$. Details of mutual information computation can be found in 12 . The Newton method for updating the parameter sets is $W^{(t+1)}=W^{(t)}-\gamma Q\left[R^{(t)}\right]^{-1} \nabla R^{(t)}$, where $W^{(m)}=\left[E_{s}^{(m)}, E_{d}^{(m)}\right]^{T}, Q\left[R^{(t)}\right]$ is the Hessian of the error-function and $\nabla R$ its gradient. Here we use $E_{s}^{(0)}=0.5$ and $E_{d}^{(0)}=0.5$ as it is valid for the parameter coefficient values of the chosen reflectance models.

The two zenith angle estimates $\theta_{s}$ and $\theta_{d}$ are ideally identical as they represent the same object. However, they differ from each other due to shadows and texture in the input images and the limited capacities of the chosen reflectance models. Here for simplification we follow the constraint that the actual surface normal $\theta$ is the mean value of the two estimates, which is $\theta=\left(\theta_{s}+\theta_{d}\right) / 2$.

Color Estimation: From the dichromatic reflection model 10, each color vector $\left(I_{R}, I_{G}, I_{B}\right)^{T}$ is determined by a linear combination of specular reflection $\left(I_{R s}, I_{G s}, I_{B s}\right)^{T}$ and diffuse reflection $\left(I_{R d}, I_{G d}, I_{B d}\right)^{T}$, which is written as

$$
\left(\begin{array}{c}
I_{R} \\
I_{G} \\
I_{B}
\end{array}\right)=k_{s}\left(\begin{array}{c}
I_{R s} \\
I_{G s} \\
I_{B s}
\end{array}\right)+k_{d}\left(\begin{array}{c}
I_{R d} \\
I_{G d} \\
I_{B d}
\end{array}\right) .
$$

The weights $k_{s}$ and $k_{d}$ depend only on the geometry of the objects in the input images, and we only focus on the color vector of diffuse reflection as it represent 
the hue properties of objects. We also assume that the estimated color vector is normalised for simplification. Using the diffuse reflectance component estimate $I_{d}$ we can obtain the corresponding values of three color channels as $I_{R d}^{\prime}, I_{G d}^{\prime}$ and $I_{B d}^{\prime}$, thus we can have $I_{R d}, I_{G d}$ and $I_{B d}$ using the equations $I_{R d}^{\prime}=w_{d} I_{R d}$, $I_{G d}^{\prime}=w_{d} I_{G d}, I_{B d}^{\prime}=w_{d} I_{B d}$ and $\sqrt{I_{R d}^{2}+I_{G d}^{2}+I_{B d}^{2}}=1$.

\section{Estimates Analysis}

The surface properties of shape, color and reflectance are stored in the form of matrices which correspond to every pixel of input images. In this section we show how to eliminate the redundant information in the estimates and convert them into long vectors.

Reflectance: For simplification we assume the illumination and viewing direction are identical, and the reflectance properties are independent of the surface azimuth angle. The reflectance functions for each experimental objects are created by using the method described in [13. For every pixel in the image, its intensity has the corresponding value of the surface radiance function $g(\theta(x, y))=I(x, y)$. By tabulating these two values against each other, we have a dense but noisy sampling of the function $g$. Then we bin the values of $g(\theta(x, y))$ into $\eta$ bins whose width is $\tau$. Let $\Gamma_{i}=(x, y) \mid(i-1) \tau \leq \theta(x, y)<i \tau$ be the set of pixels $(x, y)$ for which $\theta(x, y)$ falls into the $i$ th bin. For each bin we find the median value of $g: h(i)=\underset{(x, y) \in \Gamma_{i}}{\operatorname{median}} I(x, y)$. Then the reflectance function is stored as a long vector $(h(1), \ldots, h(\eta))$.

Color: The hue value in HSI color representation ranges from 0 to 360 degrees, and each degree stands for a specific color which can be converted from RGB triplet. The aim here is to compute hue values from every pixel in the image, and create hue distribution vector which is the histogram of all hue values. We convert RGB values to hue by using the equations described in 14, which are

$$
\begin{gathered}
{\left[\begin{array}{l}
Y \\
C_{1} \\
C_{2}
\end{array}\right]=\left[\begin{array}{ccc}
1 / 3 & 1 / 3 & 1 / 3 \\
1 & -1 / 2 & -1 / 2 \\
0 & -\sqrt{3} / 2 \sqrt{3} / 2
\end{array}\right]\left[\begin{array}{l}
I_{R d} \\
I_{G d} \\
I_{B d}
\end{array}\right],} \\
\text { Hue }= \begin{cases}\arccos \left(C_{2} / \sqrt{C_{1}^{2}+C_{2}^{2}}\right), & C_{1} \geq 0 ; \\
2 \pi-\arccos \left(C_{2} / \sqrt{C_{1}^{2}+C_{2}^{2}}\right), & C_{1}<0 .\end{cases}
\end{gathered}
$$

Shape: Wu et al. 9] developed a framework which uses methods based on principal geodesic analysis (PGA) to extract surface shape features of facial needlemap recovered by shape from shading (SFS), and implement gender classification based on the estimates. PGA is a generalisation of PCA which can be applied to feature extraction for 3D shape analysis. However, there are two drawbacks. Firstly, as SFS is proved to be an ill-posed problem the method can not be used for general objects. Secondly, there is a requirement that the input images 
should be fully-aligned and subjects have no boundaries. Here we present a novel method to solve these problems.

Denote $\theta$ as the data matrix containing zenith angle information obtained in the previous section. We recover a height map $B$ using shapelets method [15] without the need of azimuth angle estimates. The values of height are represented in Cartesian coordinate, which can lead to a loss of information and poor performance in feature extraction. To overcome this problem we embed the points on a spherical manifold system, which represents the size of object and shape variations in a convenient way. Suppose the center point of the object locates at $\left(x_{0}, y_{0}, z_{0}\right)$, for any point in the surface whose location is $(x, y, z)$ its spherical coordinate triplet $(r, \vartheta, \varphi)$ is computed by the following equations

$$
\begin{aligned}
& r=\sqrt{\left(x-x_{0}\right)^{2}+\left(y-y_{0}\right)^{2}+\left(z-z_{0}\right)^{2}}, \\
& \vartheta=\cos ^{-1}\left[\sqrt{\left(x-x_{0}\right)^{2}+\left(y-y_{0}\right)^{2}} / r\right], \\
& \varphi=\left\{\begin{array}{ll}
\cos ^{-1}\left[\left(y-y_{0}\right) / \sqrt{\left(x-x_{0}\right)^{2}+\left(y-y_{0}\right)^{2}}\right], & x \geq x_{0} \\
2 \pi-\cos ^{-1}\left[\left(y-y_{0}\right) / \sqrt{\left(x-x_{0}\right)^{2}+\left(y-y_{0}\right)^{2}}\right], & x<x_{0}
\end{array} ;\right.
\end{aligned}
$$

where $r$ is the radial distance between the surface point and the center point of object, the zenith angle $\vartheta$ and the azimuth angle $\varphi$ are the directions from $\left(x_{0}, y_{0}, z_{0}\right)$ to $(x, y, z)$. Suppose a $3 \times x y$ matrix $B^{\prime}=\left(b_{1}^{\prime}, b_{2}^{\prime}, b_{3}^{\prime}\right)$ as the height map presented in spherical coordinate, in which the three rows represent the values of $r, \vartheta$ and $\varphi$ respectively. We create a $2 \mathrm{D}$ radius distribution matrix $T$ in which the row is for azimuth angle ranges in $[0,2 \pi]$ while as the column is for zenith angle lies in the closed interval $[0, \pi / 2]$. We bin the values of $B^{\prime}$ into $30 \times 120$ sqaureshaped windows of size $3 \times 3$. Let $w(x, y)$ in $B^{\prime}$ be the set of rows that follows $w(x, y)=\left\{(x, y) \mid 3(x-1) \leq h_{1}^{\prime}<3 x, 3(y-1) \leq h_{2}^{\prime}<3 y\right\}$, for each set we find the median value of $b_{3}^{\prime}(w(x, y))$ and stored in $T$ as $T(x, y)=\operatorname{median}_{(x, y) \in w(i, j)} B^{\prime}(x, y)$.

The values in $T$ which the corresponding set is empty with $B^{\prime}$ in their range are set to be 0 . The $2 \mathrm{D}$ matrix $T$ is then converted to be long vectors for the feature extraction.

\section{Feature Extraction}

From a set of sample data, Principal Component Analysis (PCA) aims to find a linear subspace which maximises the variance of the projected data. It is widely applied in the fields of dimensionality reduction and feature extraction. However, the projections calculated by PCA usually are not those that best separate the data into distinct classes. Wu et. al [9] proposed several weighting schemes to improve the discriminating capacity of the leading PCA eigenvectors for gender classification. Here we extend their idea to multiple class recognition.

Firstly we incorporate a pre-computed weight map into PCA, namely weighted PCA (WPCA). The weight map is a representation of the discriminating capacity for each location in the long vectors. The locations that better identify objects are assigned higher weights than the rest part. Suppose there are $m$ classes from 
the $n$ input data $X=\left[x_{1}, x_{2}, \ldots, x_{n}\right]$, and the mean vectors for each class are denoted $\left[\bar{x}_{1}, \bar{x}_{2}, \ldots, \bar{x}_{m}\right]$. The weight at location $l$ is computed as

$$
w_{l}=1-\exp \left\{-\left[\sum_{i=1}^{m}\left(\bar{x}_{i, l}-\bar{x}_{l}\right)\right]^{2} / m^{2}\right\},
$$

where $\bar{x}$ is the mean vector of all the data in $X$. By making use of the mean vectors, the constructed weight map is less influenced by the difference in the data of the same class. Next, the weight map $W=\left[w_{1}, w_{2}, \ldots, w_{n}\right]^{T}$ is multiplied component-wise with each long vector in $X$, and we have the set of weighted data $X^{\prime}=\left[W . * x_{1}, W . * x_{2}, \ldots, W . * x_{n}\right]$, where $*$ denotes componentwise matrix multiplication. We apply Singular Vector Decomposition (SVD) to $X^{\prime}$ which gives $X^{\prime}=C S V^{T}$, where $C$ is the left eigenvector matrix represented as feature components, $S$ the diagonal matrix of singular values, and $V$ the right eigenvector matrix which consists of $d$ dimensional feature vectors.

We also extend the above approach by learning the weight map in a supervised way, which is termed supervised weighted PCA (SWPCA). Suppose we apply PCA to a data set $X=\left[x_{1}, x_{2}, \ldots, x_{n}\right]$, and obtain the leading $k$ eigenvectors $\Phi=\left[e_{1}, \ldots, e_{k}\right]$ and the corresponding eigenvalues $\Lambda=\left[\lambda_{1}, \ldots, \lambda_{k}\right]$. The PCA feature vector for data $x \in X$ is $v=\Phi^{T} x$, which can be expressed componentwise as

$$
v_{i}=\sum_{l=1}^{d} \Phi_{i l}^{T} x_{l}
$$

where $d$ is the dimension of data $x, \Phi_{i}$ denotes the $i$ th eigenvector, and $\Phi_{i l}$ is its value at the location $l$. SWPCA extends the above component-wise feature extraction by incorporating a weight map as $v_{i}^{S W}=\sum_{l=1}^{d} \Phi_{i l}^{T} w_{l} x_{l}$, where $w_{l}$ is the weight at the location $l$. Because the weight map has a large absolute value in class-discriminating regions, SWPCA increases the influence of class-discriminating regions over the extracted features and decreases that of the non-discriminating regions. Suppose there are $m$ classes in the data set $X=\left[x_{1}, x_{2}, \ldots, x_{n}\right]$, which are denoted class $i, i=1, \ldots, m$. The weight map is initialized as the one used in WPCA, and is optimised by minimising an error function,

$$
\xi=\sum_{i=1}^{m} \sum_{j \in \text { class }_{i}} \frac{D\left(v_{j}, \bar{v}_{i}\right)^{2}}{D\left(v_{j}, \overline{\tilde{v}}_{i}\right)^{2}}
$$

where $v_{j}$ is the WPCA feature vector (normalized by eigenvalues $S$ ) of data $x_{j} \in X, \bar{v}_{i}$ is the mean feature vector for class $s_{i}$, and $\overline{\tilde{v}}_{i}$ is mean of the data not belonging to class $_{i}$. Function $D$ calculates the Euclidean distance between the feature vectors. Substituting Equation (10) into Equation (11), we have $\xi$ to update the weight map $W$ using $W^{(t+1)}=W^{(t)}-\nabla \xi\left(W^{(t)}\right)$. We use gradient descent method to optimise each $w_{l}$ in the weight map. 


\section{Experiment Results}

In this section we present experimental evaluations of our framework for surface characteristics estimation. During the acquisition we placed a vertical polarisation filter in front of a collimated light source, so that the object are illuminated by polarised light in the direction of the camera (frontal illumination). There is also a polariser in front of the camera, which can be rotated and change the intensities of input images following the equation of Transmitted Radiance Sinusoid (TRS) [16.

There are 35 fruits in 7 different categories for the experiments, and there are 5 objects in each class. The experimental objects include red and green apples, oranges, pears, tomatoes, lemons and apricots. The results of reflectance functions and hue distributions for all inputs are shown in Fig 1 The reflectance information of fruits in different classes is hard to distinguish, as it is easily

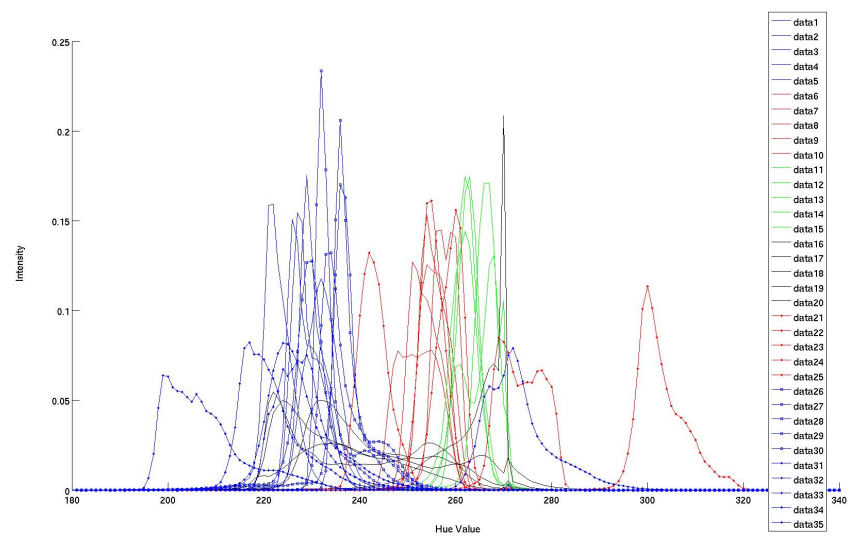

(a) Hue Distribution

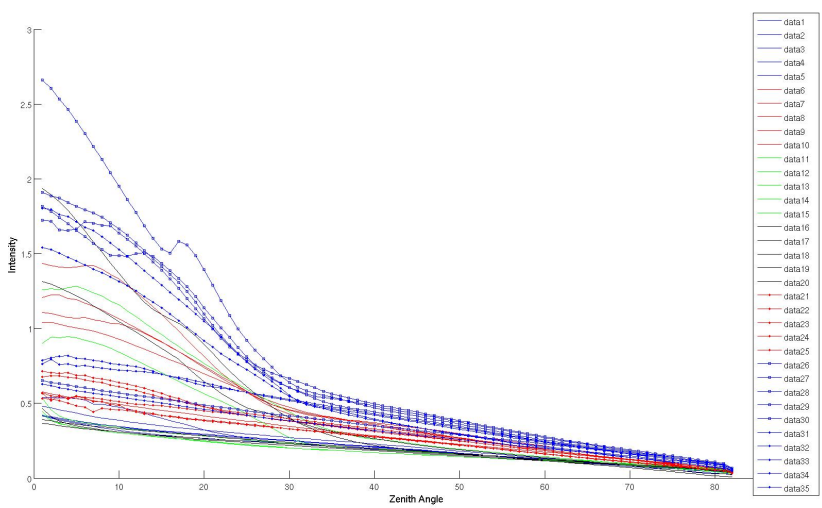

(b) Reflectance Function

Fig. 1. The estimated hue distribution and reflectance functions for 35 fruit objects 


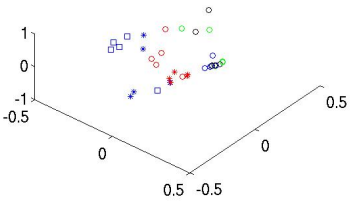

(a) PCA of Ref.

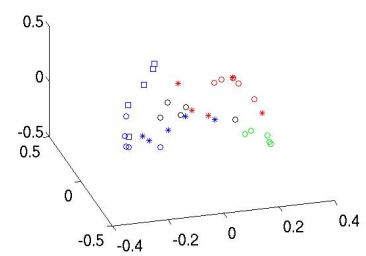

(d) PCA of Hue

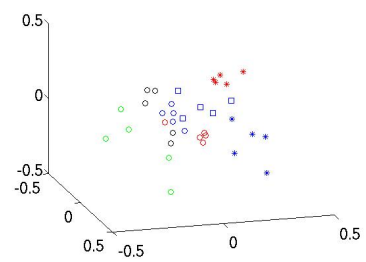

(g) PCA of Shape

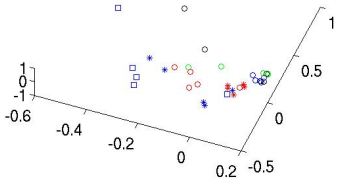

(b) WPCA of Ref.

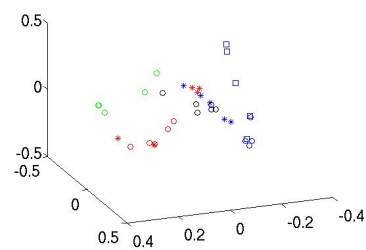

(e) WPCA of Hue

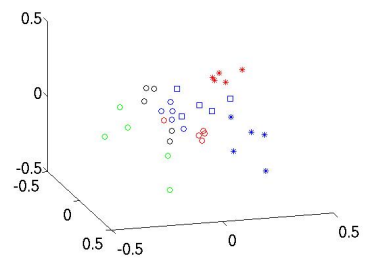

(h) WPCA of Shape

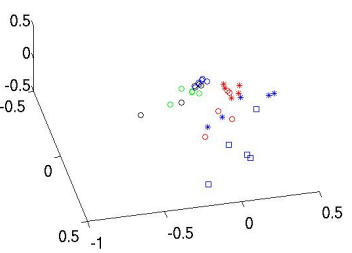

(c) SWPCA of Ref.

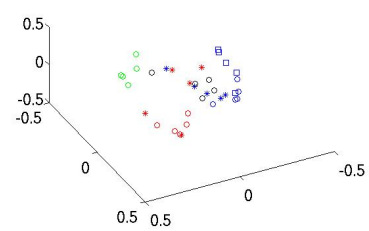

(f) SWPCA of Hue

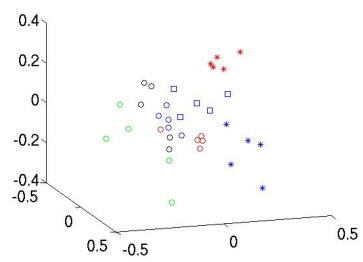

(i) SWPCA of Shape

Fig. 2. The three feature extraction methods used for three characters of fruit surface appearance

influenced by dirts such as dusts and oil. The hue distributions are easier to recognise as the light source conditions are identical for all inputs, however some objects in different classes have similar or even identical colors. Therefore using only color information cannot discriminate fruits accurately, but the properties of shape and reflectance should also be considered.

In Fig 2 we show the feature extraction results using the three methods, i.e. PCA, WPCA and SWPCA. From the figure, the features extracted using SWPCA are better separated by different classes, and are more concentrated within the same class than those extracted using the other two methods. It is also clear that using techniques such as nearest neighbour or SVM the fruit classification results can be much improved when considering the three properties simultaneously. This is one of the topics for future research.

\section{Conclusion}

In this paper we provide a novel framework for obtaining shape, color and reflectance information using the polarisation techniques and then uses three feature extractions methods on the estimates. We demonstrate experimentally that 
the proposed methods are robust and reliable, which can be applied in object recognition and classification. Future research will explore these applications.

\section{References}

1. Gehler, P., Nowozin, S.: On feature combination for multiclass object classification. In: International Conference on Computer Vision, pp. 221-228 (2009)

2. Nicodemus, F.E., Richmond, J.C., Hsia, J.J., Ginsberg, I.W., Limperis, T.: Geometrical considerations and nomenclature for reflectance. Nbs Monograph 160, 1-52 (1977)

3. Li, H., Foo, S.C., Torrance, K.E., Westin, S.H.: Automated three-axis gonioreflectometer for computer graphics applications. In: Society of Photo-Optical Instrumentation Engineers (SPIE) Conference Series, vol. 5878, pp. 221-231 (2005)

4. Debevec, P., Hawkins, T., Tchou, C., Duiker, H.P., Sarokin, W., Sagar, M.: Acquiring the reflectance field of a human face. In: Proceedings of the 27th Annual Conference on Computer Graphics and Interactive Techniques, SIGGRAPH 2000, pp. $145-156(2000)$

5. Ma, W.C., Hawkins, T., Peers, P., Chabert, C.F., Weiss, M., Debevec, P.: Rapid acquisition of specular and diffuse normal maps from polarized spherical gradient illumination. Rendering Techniques (9), 183-194 (2007)

6. Nayar, S.K., Fang, X.S., Boult, T.: Separation of Reflection Components using Color and Polarization. International Journal of Computer Vision 21(3), 163-186 (1997)

7. Wolff, L.B., Boult, T.E.: Constraining object features using a polarization reflectance model. IEEE Transactions on Pattern Analysis and Machine Intelligence 13, 635-657 (1991)

8. Zhang, L., Hancock, E., Atkinson, G.: Reflection component separation using statistical analysis and polarisation. IbPRIA, 476-483 (2011)

9. Wu, J., Smith, W.A.P., Hancock, E.R.: Gender discriminating models from facial surface normals. Pattern Recognition 44(12), 2871-2886 (2011)

10. Shafer, S.A.: Using color to separate reflection components. Color Research \& Application 10(4), 210-218 (1985)

11. Torrance, K.E., Sparrow, E.M.: Theory for off-specular reflection from roughened surfaces. Journal of the Optical Society of America 57(9), 1105-1112 (1967)

12. Zhang, L., Hancock, E. R.: Robust Shape and Polarisation Estimation Using Blind Source Separation. In: Real, P., Diaz-Pernil, D., Molina-Abril, H., Berciano, A., Kropatsch, W. (eds.) CAIP 2011, Part I. LNCS, vol. 6854, pp. 178-185. Springer, Heidelberg (2011)

13. Smith, W., Hancock, E.R.: Recovering face shape and reflectance properties from single images. In: International Conference on Automatic Face \& Gesture Recognition, pp. 1-8 (2008)

14. Zhang, C., Wang, P.: A new method of color image segmentation based on intensity and hue clustering. In: International Conference on Pattern Recognition, vol. 3, pp. 613-616 (2000)

15. Kovesi, P.: Shapelets correlated with surface normals produce surfaces. In: International Conference on Computer Vision, vol. 2, pp. 994-1001 (2005)

16. Atkinson, G.A., Hancock, E.R.: Recovery of surface orientation from diffuse polarization. IEEE Transactions on Image Processing 15(6), 1653-1664 (2006) 